\title{
The Role of Ultrasound Cervical Assessment in Prediction of Preterm Labor
}

\author{
Mohamed Atef Behery $^{\mathrm{a}}$, Sherin Shazly ${ }^{\mathrm{b}}$
}

a

Department of Obstetrics and Gynecology, ART unit Al Azhar University, Egypt, ${ }^{\mathrm{b}}$ Department of obstetrics and Gynecology, Faculty of Medicine, Zagazig University, Egypt.

Correspondence to:

Mohamed Atef Behery, Department of Obstetrics and Gynecology, ART unit Al Azhar University, Egypt

Email:

behery133@hotmail.com

Received: 4 April, 2020

Accepted: 22 June, 2020

\begin{abstract}
:
Background: The objective of the study was to verify whether ultrasound vaginal cervicometry, performed in the midtrimester of gestation, can effectively predict preterm delivery. This document is a guideline for effective use of transvaginal ultrasound in assessment of such risk. Material/Methods: 247 singleton pregnancies subdivided into 2 groups (group $\mathbf{1}=53$ cases and include the patient with cervical length $<3 \mathrm{~cm}$ and or funneling of the cervix was detected at the time of scanning and group2= 194 patients in which no risk factor regarding cervicometry was detected) were prospectively studied from the middle of gestation until delivery. In the 18th-20th week of gestation we performed ultrasound vaginal cervicometry to determine the length of the cervix, judge the shape of the internal os, and evaluate the cervix as normal or abnormal on the basis of these parameters. With the aid of one dimensional and multidimensional analysis, we tested the dependence of completed weeks of gestation and preterm delivery on the results of ultrasound cervicometry. Results: the preterm delivery was detected in 42 patients (79\%) in group1 and was 8 patients (4.1) \% in group2 with a statistically significant difference $(\mathrm{p}<0.001)$. Conclusion: The
\end{abstract} implementation of ultrasound vaginal cervicometry at 18-20 weeks in the prenatal screening program can lead to effective and early detection of women with a significantly increased risk of preterm delivery.

Key Words: Cervical length, Funneling, preterm delivery (PTD), ultrasound 



\section{Introduction}

Although the causes of preterm birth are multiple, short cervical length (CL), detected via transvaginal ultrasonography between 20 and 24 weeks, has been implicated in the mechanisms that lead to preterm birth. Short CL has been found in approximately $0.5 \%-$ $3.0 \%$ of asymptomatic singleton pregnant women. $(\underline{1}, \underline{2})$

Many studies reported that maternal characteristics such as an obstetric history, prior cervical excisional procedure, and maternal weight and height, may confer a high risk of preterm delivery (PTD). $(\underline{3}, \underline{4})$

However, clarifying this issue has important clinical implications for patient counseling, appropriate screening, and developing preventive and treatment strategies for preterm birth. (ㅁ)Preterm birth is the leading cause of perinatal mortality and morbidity. (ㅁ$)$

The mortality rate among prematurely delivered neonates represents up to $70 \%$ of total perinatal and early neonatal mortality. Morbidity and the probability of permanent physical or mental handicaps are also greater in these children. Despite advances in perinatal care, the incidence of preterm birth continues to rise primarily due to increased multiple pregnancies as a result of assisted reproduction. (ㄱ)
Despite low sensitivity and predictive value, cervical length is the only predictor for which an effective intervention is potentially available. (ㅁ)

Tocolytics prolong minimally pregnancy once preterm labor has begun and are associated with significant undesirable maternal, fetal, and neonatal consequences. (ㅁ)

To address the prematurity problem, identification of those at increased risk is an important objective.

Increased preterm birth risk is associated with many variables, including socioeconomic marginalization, previous preterm birth, cervical 'incompetence,' smoking, and nonmedical drug use. Pregnancy complications that increase likelihood of preterm birth include multiple gestation, polyhydramnios, vaginal bleeding, certain uterine anomalies, and excessive uterine contractions. Despite these important associations, maternal risk scoring fails to detect up to 70 percent of infants who are delivered spontaneously before term. (10). Other screening strategies that have been suggested include measuring of biochemical markers of inflammation and culturing for infections. ( $\underline{11})$

The expense of specialized care for preterm neonates is another factor prompting a search 
for an effective program of prevention. One of the methods contributing to the prevention of prematurity is ultrasound (US) cervicometry.

This method was put into practice toward the end of the 70s. However, wider clinical applications became possible with the appearance of high frequency vaginal transducers during the $90 \mathrm{~s}$, when it was repeatedly proved that transvaginal cervicometry is more precise than transabdominal cervicometry and examination of the cervix by palpation $(\underline{6}, \underline{7})$.

In addition transvaginal ultrasound enables us to objectively examine the supravaginal part of the cervix, especially the shape of the internal os and the lower uterine segment (10). US cervicometry is an objective examination of the uterine cervix, based on determining the length of the closed portion of the cervix and the shape of the internal os.

Preterm labor has major perinatal complications; so, the preventive measures are pivotal that is why we conducted his study, to evaluate the value of midtrimestric ultrasound in prediction of preterm delivery.

\section{Material and methods}

Two hundreds forty seven singleton pregnancy patients underwent ante natal care at Zagazig University Hospital in the period from March 2016 to September 2017 with the following criteria

\section{Inclusion criteria,}

a- Pregnant in singleton pregnancy (PTD)

b- Age $<40$

c- $\mathrm{BMI}<30$

d- Between 18 and 20 weeks gestation

e- With no cervical cerclage

f- No chronic medical diseases like hypertension or diabetes

exclusion criteria

a- Patients with history of preterm delivery

b- Previous uterine surgery

c- Multiple gestations.

The included patients were divided into 2 groups (group $1=53$ patients with cervical length $<3 \mathrm{~cm}$ and or funneling of the cervix was detected at the time of scanning and group2= 194 patients with no risk factor according to cervicometry) and were prospectively studied from the middle of gestation until delivery. All the studied women were submitted to vaginal ultrasound cervicometry, which we used to determine the length of the cervix, judge the shape of the internal os, and evaluate the cervicometry as normal or abnormal on the basis of these parameters. With the aid of one dimensional and multi-dimensional analysis, we tested the dependence of completed weeks of gestation and preterm delivery on the results of ultrasound cervicometry. 


\section{Statistical analysis}

Quantitative data was analyzed by parametric statistics (student t test was used) while Chisquare test was used for analysis of categorical data using SPSS version 20, (IBM Company New York, USA).KolmogorovSeminirov test was used for testing normality of the different variables and $\mathrm{P}$ - value was considered significant at a level $\leq .05$. The receiver operating characteristics (ROC) curve was used to detect the sensitivity of the cervicometry in prediction of PTD. Logistic regression analysis was used to determine the effect of the different factors on the occurrence of PTD.

\section{Results:}

In group1 (53 cases), 42 cases (79\%) patients underwent PTD, while in group2 (195 cases), PTD occurred in 8 cases $(4.1 \%)$ with a significant difference between both groups $(\mathrm{p}<0.001)$ Table 1.

In group1, twenty four women (45\%) had a cervical length less than $30 \mathrm{~mm}$. Only three women (6\%), had a cervical length less than $20 \mathrm{~mm}$ and all of them delivered prior to the 34th week of gestation. High-risk funneling of the internal os (at rest or during increased intra-abdominal pressure) was found in 26 women (49\%). The average gestational age for group1 is 36 weeks gestation and for group2, it is 39.12 weeks with no significant difference between both groups $(\mathrm{p}=0.102)$, table 1.

Out of 279 women, who met the inclusion criteria, 247 had completed the study while 32 cases were dropped from the study due to social and noncompliance factors.

The frequency of births prior to the end of the 37th week (PTD) in group1 was 79\% (42 women).

The frequency of births prior to the end of the 37 th week (PTD) in group2 was $4.1 \%$ (8 women). (figure1)

By binary logistic regression analysis, the age $(\mathrm{OR}=0.83,95 \%$ CI $0.54-1.28$ and $\mathrm{P}>0.05)$ and $\mathrm{BMI}(\mathrm{OR}=0.88,95 \% \mathrm{CI} 0.61-1.26$ and $\mathrm{P}$ $>0.05$ ) are not good predictor for preterm delivery incidence. On the other hand the cervical length is a good predictor $(\mathrm{OR}=0.49$ ,95\% CI 0.12-0.89 and $\mathrm{P}<0.05)$,i.e., $51 \%$ increase in PTD in patients with cervical length $<3 \mathrm{~cm}$ measured at 18-20 weeks of gestation if compared to patients with cervical length $>3 \mathrm{~cm}$ measured at the same gestational age (table 2).

Receiver operating characteristic (ROC) curve was done to detect the sensitivity of midtrimestric ultrasound cervicometry in prediction of PTD, the results showed that at a cut off value of $<3.5 \mathrm{~cm}$, the vaginal ultrasound was highly accurate in prediction of PTD with a sensitivity of $94 \%$. Area under 

the curve (AUC) was 0.88., and p-value $<0.05$ (figure 2 and table 3 ).

Table (1): shows the comparisons between both groups as regard cervical length, frequency of funneling and PTD.

\begin{tabular}{lccc}
\hline Parameter & $\begin{array}{c}\text { Group 1 (with risk } \\
\text { factor) } \mathbf{N O = 5 3}\end{array}$ & $\begin{array}{c}\text { Group 2( no risk factor) } \\
\text { NO=194 }\end{array}$ & p-value \\
\hline $\begin{array}{l}\text { Cervical length } \\
(\text { mean } \pm \text { SD) }\end{array}$ & $3.1 \pm 0.82$ & $4.6 \pm 0.43$ & $<0.0001$ \\
Funneling & & & \\
(frequency) & $26 / 53(49 \%)$ & $0 / 194(0 \%)$ & $<0.0001$ \\
PTD (frequency) & $42 / 53(79 \%)$ & $8 / 194(4.1 \%)$ & $<0.001$ \\
Gestational age & $36 \pm 3.2($ mean \pm SD) & $39.12 \pm 4.5$ & 0.102 \\
\hline
\end{tabular}

Table (2): Binary Logistic Regression Analysis of age, BMI,and cervical length as Predictors of PTD.

\begin{tabular}{cccc}
\hline Variables & OR & $\mathbf{9 5 \%}$ CI & P-value \\
\hline Age & 0.83 & 0.54 to 1.28 & $>0.05$ \\
BMI & 0.88 & 0.61 to 1.26 & $>0.05$ \\
Cervical Length & 0.49 & 0.12 to 0.89 & $<0.05$ \\
\hline
\end{tabular}

Table (3): Receiver-operating characteristic (ROC) curve analysis for prediction of preterm labor using cervical length as a predictor.

\begin{tabular}{lllllll}
\hline ROC index & $\begin{array}{l}\text { AUC } \\
\text { (area } \\
\text { curve) }\end{array}$ & under & 95\% CI & P-Value & $\begin{array}{l}\text { Best Cut off Value } \\
\text { (prediction probability) }\end{array}$ & $\begin{array}{l}\text { Sensitivity of } \text { test } \\
\text { Specificity } \\
\text { the test }\end{array}$ \\
\hline Cervical length & 0.822 & $0.744-0.899$ & $<0.001$ & $<3.5 \mathrm{~cm}$ & $94 \%$ & $64 \%$ \\
\hline
\end{tabular}




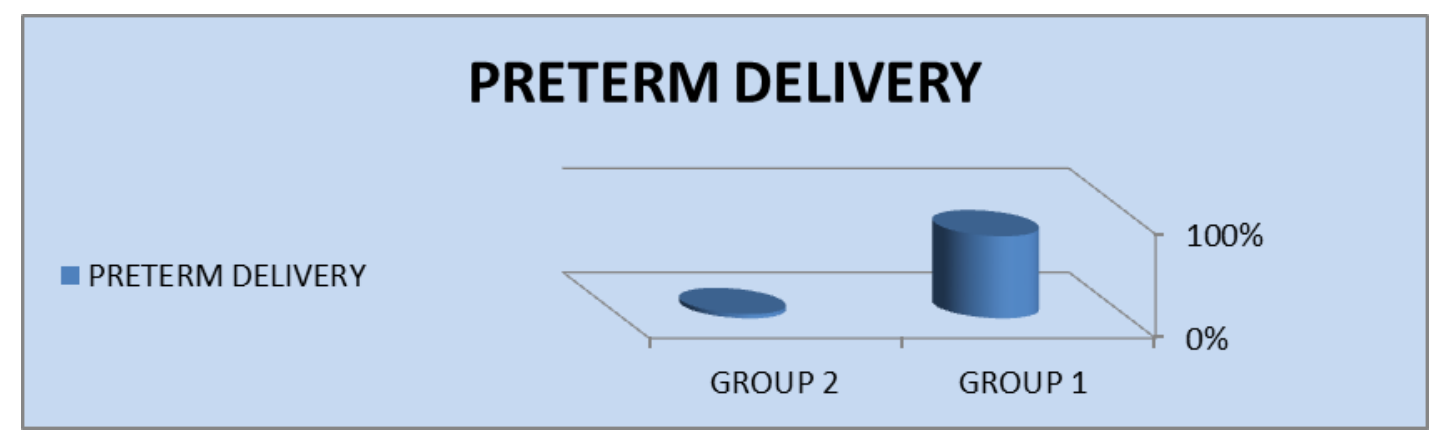

Figure (1): The percentages of cases with preterm labor among both groups.

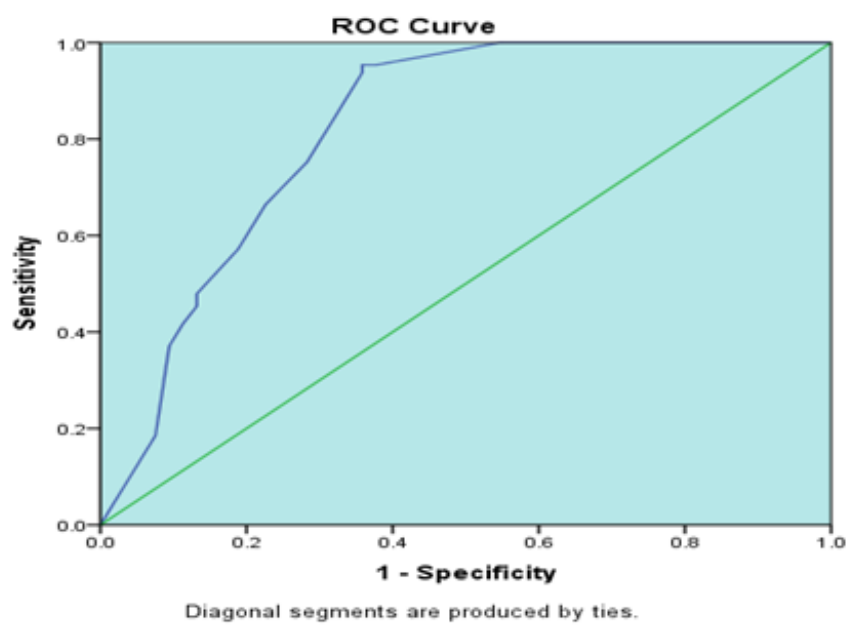

Figure (2): Receiver operating characteristics curve showes the accuracy of cervical length measurement in prediction of preterm labor.

\section{Discussion}

At present, the most commonly used definition of preterm delivery is that of the WHO: delivery between the 22nd and 37th completed week of gestation (11).

Many studies showed that $1.0 \%$ of women had a cervical length $15 \mathrm{~mm}$ or less at 22 to 24 weeks and other studies noted that $2.3 \%$ of women had similar cervical lengths. The low incidence of cervical shortening limits the utility of this screening test. These data correlate with the results reported by other authors $(\underline{12}, \underline{13})$.

One of the most wide-ranging studies dealing with cervicometry focused on the dependence of preterm delivery on the length of the cervix in the 24th week of gestation .The average cervical length at this gestational age was $35 \mathrm{~mm}$. With decreasing cervical length, the relative risk of prematurity significantly increased (14). 
Heath et al. found the relative risk of delivery prior to the33rd week of gestation to be 46.2 in women whose cervical length was less than $15 \mathrm{~mm}$. The relative risk of prematurity in the case of fetal fibronectin positivity was 8.1 (15).

The average cervical length in group1was 3.1 $\mathrm{mm}$ while it was $4.6 \mathrm{~mm}$ in group2 with a significant difference between both groups $(p=0.021)$. The most significant indicator distinguishing women with preterm delivery from those who delivered at term was the overall (abnormal) cervicometric result.

The abnormal shape of the internal os (funneling) is significant in predicting this result. In our opinion, evaluating the lower uterine segment and the quality of the internal cervical os is mostly important. Inspite these factors, we should consider the added influence of increased intra-abdominal pressure.

In agreement with this study, some authors considered abnormal shape of cervix as a very significant in prediction of PTD $(\underline{16}, \underline{17})$.

At present, prediction and screening of preterm delivery is still not overly effective. In recent years, the incidence of prematurity has not decreased significantly even in countries with highly sophisticated health care. Regardless of the promising results of many studies, ultrasound cervicometry has not yet become a routine part of prenatal care $(\underline{6}$, $\underline{12}, \underline{13})$.

The discrepancy between studies may be related to the differences in the gestational age cutoffs used to define PTD as a primary outcome measure, sample size, standard care for asymptomatic pregnant women at high risk of preterm delivery, inclusion and exclusion criteria and whether known risk factors were uniformly adjusted in the analyses (e.g., short CL) (ㄷ) .

We did not include patients with history of PTD in the study because of the previously published data $(\underline{19}, \underline{20})$, which found that the women with a prior PTD had a shorter midtrimester CL and were more likely to have short CL than the women with no history of preterm delivery (e.g., nulliparas or women at low risk), suggesting that the association between a prior PTD and the risk of subsequent PTD may be mediated by the shortening itself of the cervix.

In addition, Soo et al suggest that an unexplained factor poses a higher risk of subsequent PTD to women with prior PTD than that associated with short CL alone .

Our results confirm the previous works, showing that CL, as measured by using transvaginal scan at mid-trimester, is inversely associated with the frequency of preterm birth $(\underline{21}, \underline{22}, \underline{23}, \underline{20})$. 
Some studies demonstrated an interaction between obstetric history and short CL in association with PTD, suggesting a synergistic detrimental effect of these 2 variables on PTD. This finding also agrees with those of a previous report in which unlike low-risk women with short CL, high-risk women with prior PTD and short CL benefit from cerclage, and this benefit increases as the CL decrease (24).

Taken together, these data could help in the successful identification of women at high risk of preterm delivery by increasing patients' acceptability of universal screening for CL and in the design of current and potential future strategies for prevention and treatment of preterm delivery

However, the anatomical picture of the uterine cervix is the etiological factor in only a relatively small portion of women at risk of preterm delivery: namely, women with cervical incompetence.

On the other hand, pregnant women with a shortened or dilated cervical canal are likely to be much more susceptible to ascendant genital infection, which underlies a major portion of preterm deliveries (14).

Below 37 weeks gestation, transvaginal cervical measurement may be valuable in avoiding unnecessary interventions based on a high positive predictive value of a cervical length less than $3.5 \mathrm{~cm}$. (sensitivity 94\%).
Since amniorrhexis at or beyond 36 weeks is managed in most circumstances, as such events are dealt with at term, transvaginal ultrasound is unlikely to influence management. Transvaginal ultrasound cervical measurement in asymptomatic women, including those at increased risk of preterm birth, is reassuring if length after 24 weeks is greater than $3 \mathrm{~cm}(\underline{18})$.

Despite the value of midtrimestric ultrasound cervicometry in prediction of PTD which is supported by many studies, the application of this method as a routine is still of debate specially with singleton pregnancy in which even the first trimester cervicometry is not used routinely in many centers.

\section{Conclusion}

Transvaginal ultrasound cervical measurement is a safe and effective technique to predict increased risk of preterm delivery in selected patients. However, routine prenatal transvaginal ultrasound screening of cervical length is not supported by available evidence. Normal results can help avoid unnecessary interventions ,but randomized trials are needed to determine whether interventions can improve maternal and neonatal outcomes, given cervical shortening. 


\section{References}

1. Romero R, Espinoza J, Kusanovic JP, Gotsch F, Hassan S, Erez O, Chaiworapongsa T, Mazor M; The preterm parturition syndrome. BJOG(2006); 113 Suppl 3: $17-42$

2. Sakai M, Shiozaki A, Tabata M, Sasaki Y, Yoneda S, Arai T, Kato K, Yamakawa Y, Saito S ;Evaluation of effectiveness of prophylactic cerclage of a short cervix according to interleukin-8 in cervical mucus. Am J Obstet Gynecol(2006); 194: 14-9.

3. Facco FL, Simhan HN.; Short ultrasonographic cervical length in women with low-risk obstetric history. Obstet Gynecol(2013); 122: 858-62

4. Sharashova EE, Anda EE, Grjibovski AM ;Early pregnancy body mass index and spontaneous preterm birth in Northwest Russia: a registry-based study. BMC Pregnancy Childbirth(2014); 14: 303

5. Soo-Hyun Cho, Kyo Hoon Park, Eun Young Jung, Jung Kyung Joo, Ji Ae Jang, and Ha-Na Yoo; Maternal Characteristics, Short Mid-Trimester Cervical Length, and Preterm Delivery J Korean Med Sci(2017); 32: 488-494.

6. Berkowitz GS, Papienik E. Epidemiology of preterm birth;Epidemiol Rev(1993); 15:414-3.

7. Joseph KS, Kramer MS,Wu Wen S,Alexander D.; Determinants of preterm birth in Canada from 1981 through 1992, and 1992 through1994. N Engl J Med(1998);339:1434-9.

8. Berghella $\mathrm{V}$; Universal cervical length screening for prediction and prevention of preterm birth. Obstet Gynecol Surv. ( 2012); 67(10):653-658

9. Consensus Statement on Preterm Birth Prevention.

Collaborative Conferenceon Preterm Birth.

Ottawa,April 1998.
10. Armson BA, Dodds L.; Prediction of preterm birth in a population ofCanadian women. Int $\mathbf{J}$ Gynecol Obste(1994) t:46(Suppl 2):93.

11. Goldenberg RL, Iams JD, Mercer BM, Meis PJ, Moawad AH, Cooper RL,et al.;The Preterm Prediction Study:The value of new vs standard riskfactors in predicting early and all spontaneous preterm births.NICHDMFMU Network.Am J Public Health(1998) ;88(2):233-8.

12. Jackson GM, Ludnir J, Bader TF; The accuracy of digital examination andultrasound in the evaluation of cervical length. Obstet Gynecol( 1992) ; 72:214-8.

13. Anderson HF.;Transvaginal and transabdominal ultrasonography of theuterine cervix during pregnancy. J Clin Ultrasound(1991);19:77-83.

14. Sonek JD, Iams JD, Blumenfeld M, Johnson F, Landon M, Gabbe S.; Measurementof cervical length in pregnancy. Comparison between vaginal ultrasonographyand digital examination. Obstet Gynecol(1990);76:172-5.

15. To MS, Skentou C, Cicero S, Nicolaides KH.; Cervical assessment at theroutine 23 weeks' scan: problems with transabdominal sonography.Ultrasound Obstet Gynecol( 2000);15(4):292-6.

16. Rosati P, Guariglia L.;Acceptability of early transvaginal or abdominalsonography in the first half of pregnancy.Arch Gynecol Obstet (2000) ;264(2):80-3.

17. Braithwaite JM, Economides DL.;Acceptability of patients of transvaginalsonography in the elective assessment of the first trimester fetus. UltrasoundObstet Gynecol(1997);9(2):76-9.

18. Iams JD, Goldenberg RL, Meis PJ, Mercer BM, Moawad A, Das A, et al;The length of the cervix and the risk of spontaneous premature delivery. $\mathrm{N}$ Engl J Med( 1996);334:567-72.

19. Heath VC, Southall TR, Souka AP, Novakov A, Nicolaides KH.; cervical length at 23 weeks of gestation: relation to demographic characteristics and 
previous obstetric history. Ultrasound Obstet Gynecol(1998); 12: 30411.

20. Miller ES, Sakowicz A, Grobman WA (2015); The association between cervical dysplasia, a short cervix, and preterm birth. Am J Obstet Gynecol; 213: 543.e14.

21. Facco FL, Simhan HN.; Short ultrasonographic cervical length in women with low-risk obstetric history. Obstet Gynecol (2013); 122: 858-62.

22. Poon LC, Savvas M, Zamblera D, Skyfta E, Nicolaides KH; Large loop excision of transformation zone and cervical length in the prediction of spontaneous preterm delivery. BJOG(2012); 119: 6928.

23. Orzechowski KM, Boelig R, Nicholas SS, Baxter J, Berghella $\mathrm{V}$;Is universal cervical length screening indicated in women with prior term birth? Am J Obstet Gyneco(2015); 1; 212: 234.e1-5

24.Owen J, Hankins G, Iams JD, Berghella V, Sheffield JS, Perez-Delboy A, Egerman RS, Wing DA, Tomlinson M, Silver R, et al; Multicenter randomized trial of cerclage for preterm birth prevention in highrisk women with shortened midtrimester cervical length. Am J Obstet Gynecol(2009); 201: 375.e1-8.

To cite this article: Mohamed Atef Behery, Sherin Shazly. The Role of Ultrasound Cervical Assessment in Prediction of Preterm Labor. BMFJ 2020; 37(3): 586-595, DOI:10.21608/bmfj.2020.27211.1245 

Original article 